\title{
Solubility of Sphene in Siliceous Melts
}

D. Flanagan ${ }^{1}$, J. C. Ayers ${ }^{1}$, C. F. Miller ${ }^{1}$, E. B. Watson², F.J. Ryerson ${ }^{3}$

1) Vanderbilt University, Department of Earth and Environmental Sciences

2) Rensselaer Polytechnic Institute

3) Lawrence Livermore National Laboratory 


\section{The importance of sphene}

- Sphene records magma evolution through compositional zoning and inclusions.

- The Zr-in-sphene thermobarometer provides estimates of magmatic temperature and/or pressure.

- Sphene can be used as a geochronometer due to relatively high $\mathrm{U}$ concentrations and $\mathrm{U} / \mathrm{Pb}$ ratios.

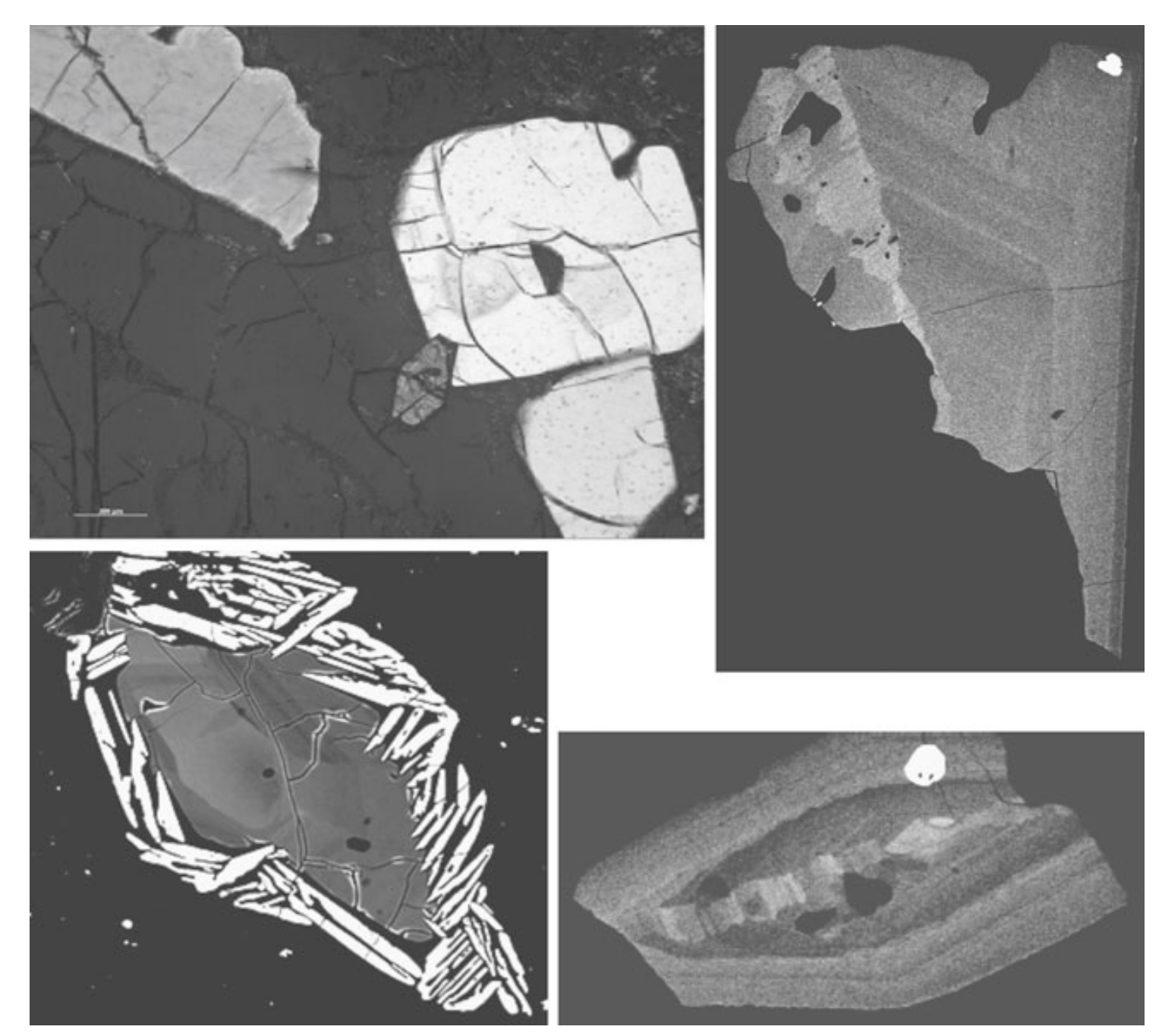

- Sphene crystallization dramatically Sphene in Highland Range rhyolites, Colombini et al. (2011) affects melt composition, especially for REE 


\section{Sphene influence on melt composition}
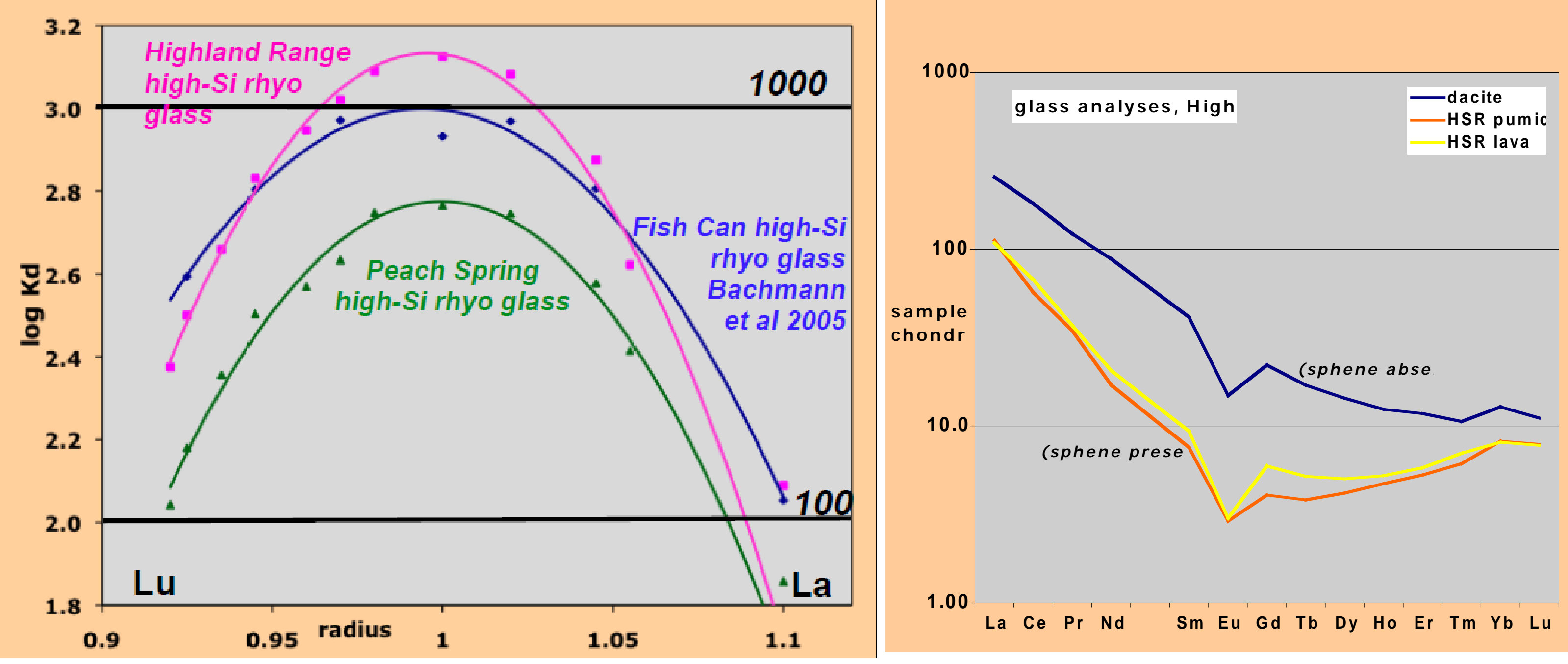


\section{Sphene stability and occurrence}

- Sphene most commonly occurs as an accessory mineral in metaluminous, high $\mathrm{fO}_{2}$, low temperature, high silica igneous rocks.

- Supported by typically low $\left(\sim 710\right.$ to $\left.780^{\circ} \mathrm{C}\right)$ sphene crystallization temperatures calculated from the Zr-in-sphene thermobarometer (Hayden et al., 2008) and by the restriction of whole-rock middle REE depletion patterns to highly evolved rhyolites and aplites.

- Peraluminous granitoids rarely contain sphene because high Al stabilizes plagioclase, and crystallization of plagioclase removes $\mathrm{Ca}$, suppressing sphene crystallization. 


\section{Sphene Solubility}

- We lack sufficient data on sphene solubility in siliceous melts to accurately predict when sphene will crystallize in a magmatic system.

- A sphene solubility equation would be useful for:

- constraining the temperatures of melts

- determining whether sphene saturation in magmatic source regions is likely

- determining when sphene can crystallize and begin to exert an influence on melt chemistry.

- We performed sphene growth and dissolution experiments to characterize the dependence of sphene solubility on pressure, temperature, and melt composition. 
Anhydrous compositions of starting materials for growth experiments

\begin{tabular}{|c|c|c|c|c|c|}
\hline & PST & PST + GSP & GSP(1) & GSP & GSP + AGI \\
\hline $\mathrm{SiO}_{2}$ (wt.\%) & 69.80 & 66.26 & 64.07 & 62.81 & 59.44 \\
\hline $\mathrm{Al}_{2} \mathrm{O}_{3}$ (wt.\%) & 11.83 & 12.93 & 14.33 & 14.05 & 15.01 \\
\hline $\mathrm{Na}_{2} \mathrm{O}$ (wt.\%) & 2.31 & 2.46 & 2.67 & 2.62 & 3.29 \\
\hline $\mathrm{K}_{2} \mathrm{O}$ (wt.\%) & 6.34 & 5.71 & 5.18 & 5.08 & 3.90 \\
\hline $\mathrm{CaO}$ (wt.\%) & 4.56 & 5.26 & 3.86 & 5.72 & 7.18 \\
\hline FeO (wt.\%) & 0.95 & 2.55 & 4.24 & 4.15 & 4.92 \\
\hline MgO (wt.\%) & 0.22 & 0.56 & 0.92 & 0.90 & 1.30 \\
\hline $\mathrm{TiO}_{2}$ (wt.\%) & 3.91 & 4.06 & 4.42 & 4.36 & 4.54 \\
\hline MnO (wt.\%) & 0.06 & 0.06 & 0.04 & 0.04 & 0.06 \\
\hline $\mathrm{P}_{2} \mathrm{O}_{5}$ (wt.\%) & 0.02 & 0.15 & 0.27 & 0.27 & 0.36 \\
\hline Total & 100.00 & 100.00 & 100.00 & 100.00 & 100.00 \\
\hline A/NK ${ }^{a}$ & 1.11 & 1.26 & 1.43 & 1.43 & 1.56 \\
\hline A/CNK ${ }^{a}$ & 0.62 & 0.65 & 0.84 & 0.70 & 0.66 \\
\hline
\end{tabular}




\section{Experimental Methods for Growth Experiments}

- Starting material compositions were metaluminous and extend from trachydacite to rhyolite.

- Experiments run in Pt and $\mathrm{Ag}_{60} \mathrm{Pd}_{40}$ capsules in graphite wells in a pistoncylinder apparatus for $48-168$ hours, $800-1000^{\circ} \mathrm{C}, \mathrm{H}_{2} \mathrm{O}$ 0-6 wt.\%, and 0.51.0 GPa.

- fO2 close to CCO buffer, between IW and QFM.

- Experiments first heated to above the liquidus, then ramped down to the run temperature.

- Run products imaged and analyzed using a Tescan Vega 3 LM SEM with Oxford X-max EDS.

- Crystal-free areas of quenched glass analyzed with 10-15 $\mu \mathrm{m}$ diameter electron beam to minimize beam damage. 


\section{Growth Experiment Run Products}

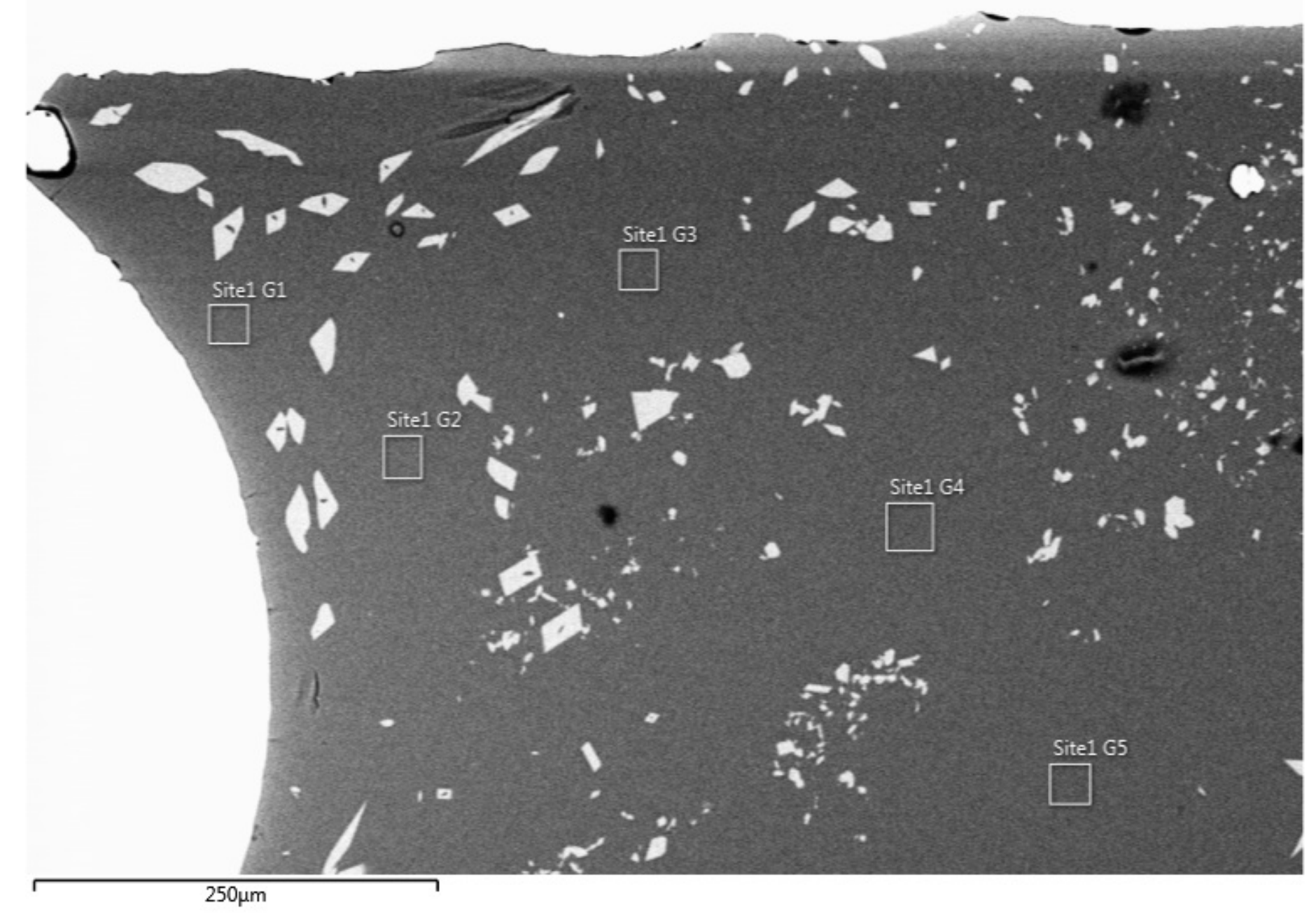

SpG.8, PST + GSP, $\mathrm{T}=900^{\circ} \mathrm{C}, \mathrm{P}=0.5 \mathrm{GPa}, \mathrm{H}_{2} \mathrm{O}=3.8$ wt. $\%$ 


\section{Growth Experiment Run Products}

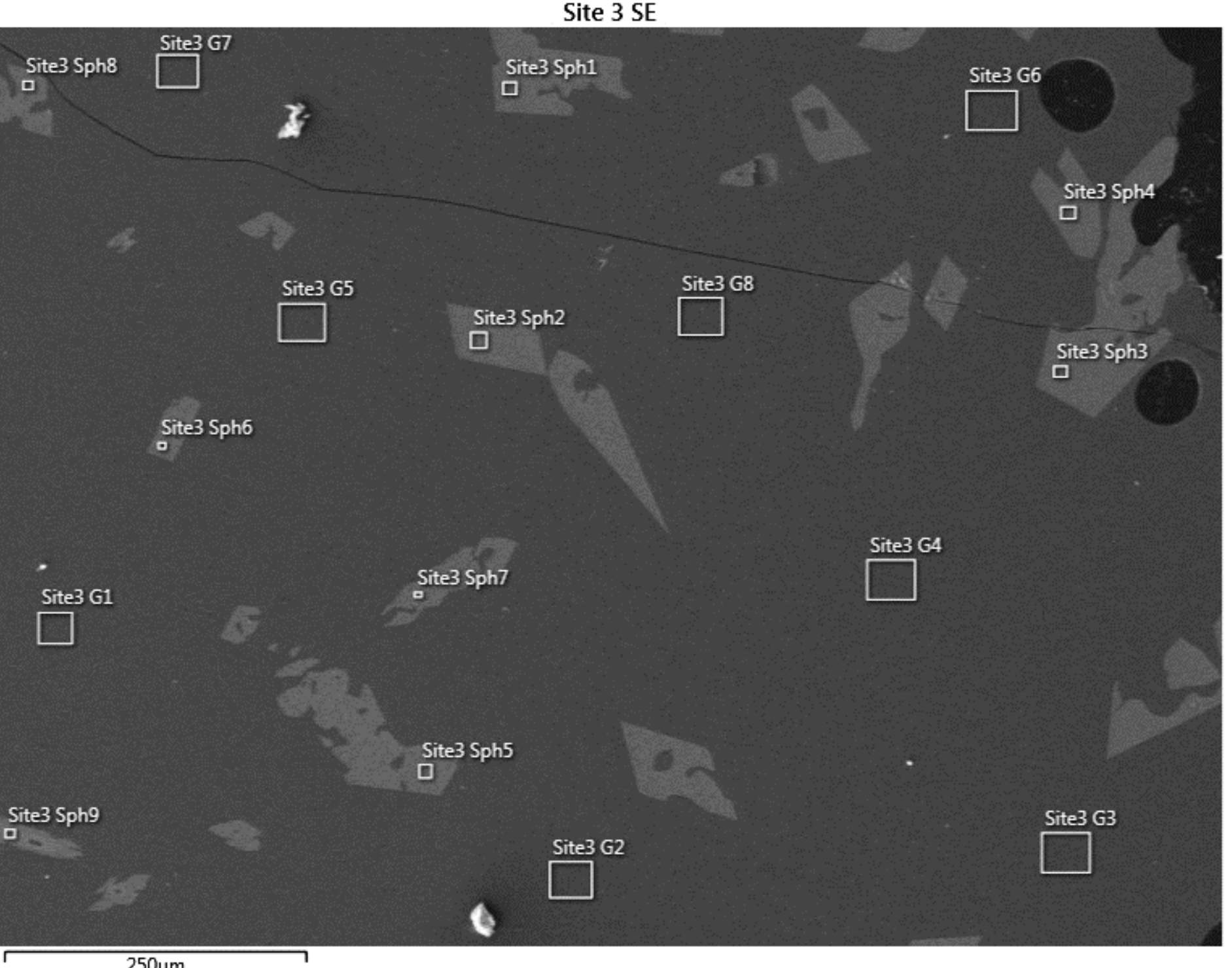

SpG.18, GSP + AGV, $\mathrm{T}=900^{\circ} \mathrm{C}, \mathrm{P}=0.5 \mathrm{GPa}, \mathrm{H}_{2} \mathrm{O}=3.7 \mathrm{wt} . \%$ 
SpG7: PST SM, $900^{\circ} \mathrm{C}, 4$ wt. $\% \mathrm{H}_{2} \mathrm{O}, 168 \mathrm{~h}$

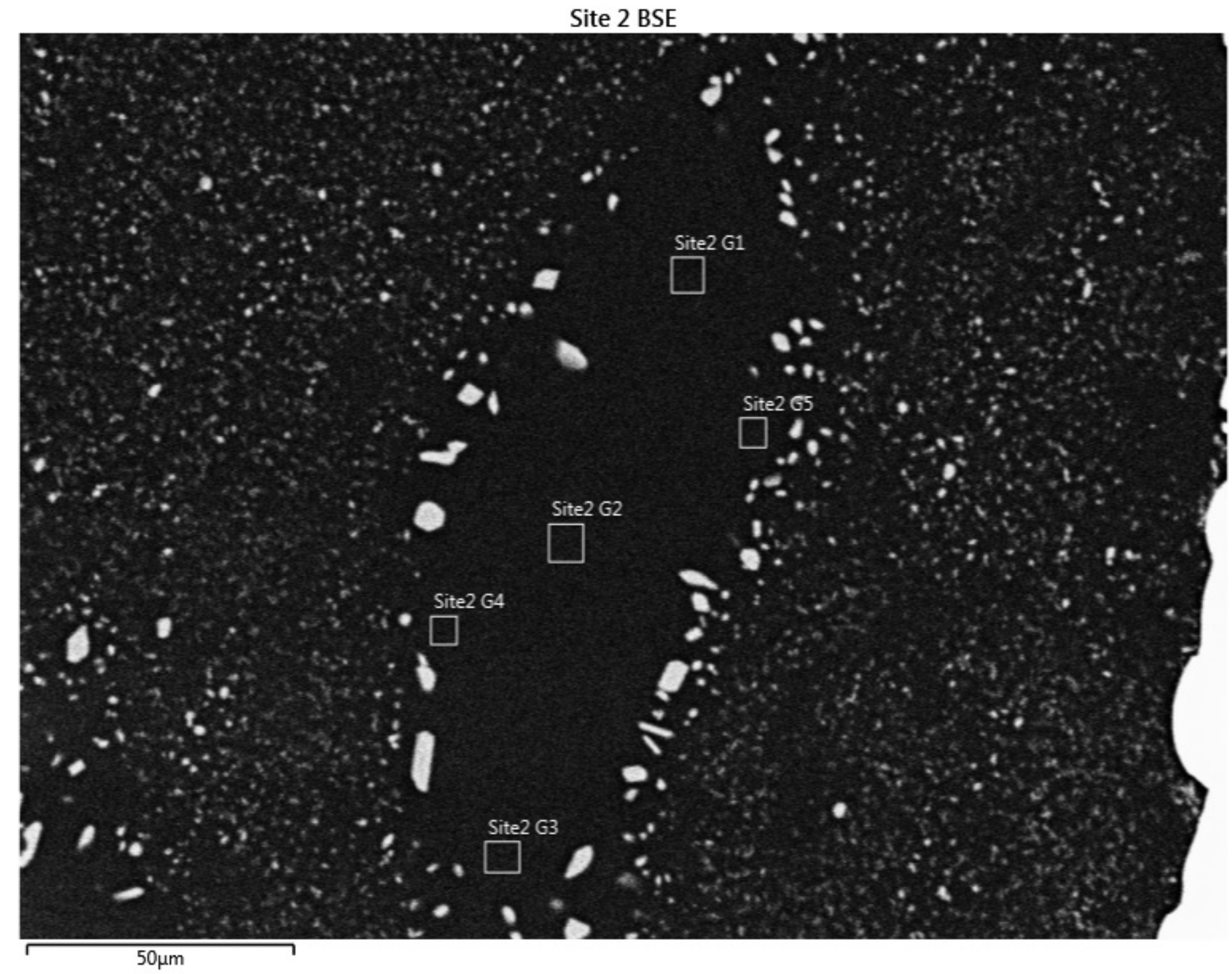




\section{Assessment of EDS Accuracy}

\begin{tabular}{|c|r|r|r|r|r|r|}
\hline Oxide & GSP nom. & GSP meas. & \% diff. & AGV-2 nom. & AGV-2 meas. & \% diff. \\
\hline$n$ & & 13 & & & 13 & \\
\hline $\mathrm{SiO}_{2}$ & 66.6 & 66.99 & $0.6 \%$ & 59.3 & 59.66 & $0.6 \%$ \\
\hline $\mathrm{Al}_{2} \mathrm{O}_{3}$ & 14.9 & 14.98 & $0.6 \%$ & 16.91 & 17.08 & $1.0 \%$ \\
\hline $\mathrm{Na}_{2} \mathrm{O}$ & 2.78 & 2.74 & $-1.4 \%$ & 4.19 & 4.26 & $1.7 \%$ \\
\hline $\mathrm{K}_{2} \mathrm{O}$ & 5.38 & 5.46 & $1.5 \%$ & 2.88 & 2.94 & $2.1 \%$ \\
\hline $\mathrm{CaO}$ & 2.10 & 2.10 & $0.2 \%$ & 5.20 & 5.12 & $-1.5 \%$ \\
\hline $\mathrm{Fe}_{2} \mathrm{O}_{3}$ & 4.90 & 4.81 & $-1.8 \%$ & 6.69 & 6.67 & $-0.3 \%$ \\
\hline $\mathrm{MgO}_{3} \mathrm{O}$ & 0.96 & 0.97 & $0.8 \%$ & 1.79 & 1.80 & $0.6 \%$ \\
\hline $\mathrm{TiO}_{2}$ & 0.66 & 0.71 & $8.0 \%$ & 1.05 & 1.06 & $1.4 \%$ \\
\hline $\mathrm{P}_{2} \mathrm{O}_{5}$ & 0.29 & 0.26 & $-10.8 \%$ & 0.48 & 0.46 & $-4.5 \%$ \\
\hline $\mathrm{Sum}$ & 98.57 & 99.02 & & 98.49 & 99.06 & \\
\hline
\end{tabular}




\section{Glass Compositions, GSP SM, $1000^{\circ} \mathrm{C}$}

\begin{tabular}{|c|c|c|c|c|c|}
\hline & GSP SM & \multicolumn{2}{|c|}{ DF-SpG.29 } & \multicolumn{2}{|c|}{ DF-SpG.33 } \\
\hline Capsule & & $\mathrm{AgP}$ & & $\mathrm{Pt}$ & \\
\hline Oxide & Conc. (wt.\%) & $\begin{array}{l}\text { Conc. } \\
\text { (wt.\%) }\end{array}$ & $1 \sigma$ & $\begin{array}{l}\text { Conc. } \\
\text { (wt.\%) }\end{array}$ & $1 \sigma$ \\
\hline $\mathrm{SiO}_{2}$ & 67.96 & 66.21 & 0.38 & 68.29 & 0.03 \\
\hline $\mathrm{Al}_{2} \mathrm{O}_{3}$ & 15.20 & 15.81 & 0.38 & 15.62 & 0.01 \\
\hline $\mathrm{Na}_{2} \mathrm{O}$ & 2.78 & 3.14 & 0.07 & 2.98 & 0.01 \\
\hline $\mathrm{K}_{2} \mathrm{O}$ & 5.54 & 5.29 & 0.14 & 5.53 & 0.01 \\
\hline $\mathrm{CaO}$ & 2.13 & 3.61 & 0.21 & 4.06 & 0.01 \\
\hline $\mathrm{FeO}(\mathrm{t})$ & 4.39 & 3.19 & 0.16 & 0.04 & 0.004 \\
\hline $\mathrm{MgO}$ & 0.98 & 0.82 & 0.04 & 1.00 & 0.004 \\
\hline $\mathrm{TiO}_{2}$ & 0.72 & 1.66 & 0.08 & 2.21 & 0.02 \\
\hline $\mathrm{MnO}$ & 0.03 & 0.04 & 0.004 & 0.03 & 0.003 \\
\hline $\mathrm{P}_{2} \mathrm{O}_{5}$ & 0.26 & 0.23 & 0.01 & 0.24 & 0.01 \\
\hline um & & 100.00 & & 100.00 & \\
\hline
\end{tabular}




\section{Dissolution Experiments: SpDis.3 Run Products}




\section{Evaluation of secondary fluorescence and precision}

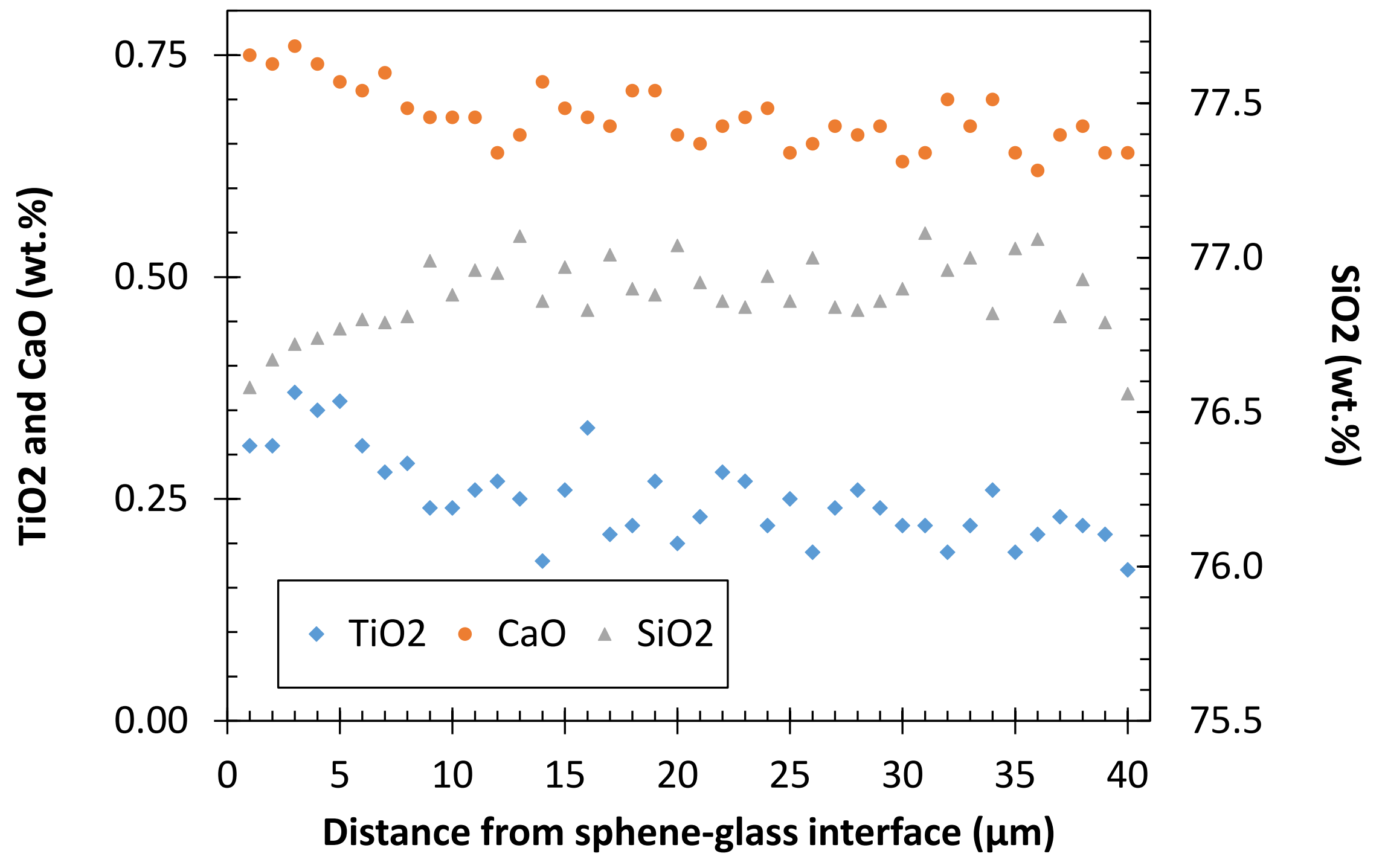




\section{SpDis.2 Diffusion Profiles}

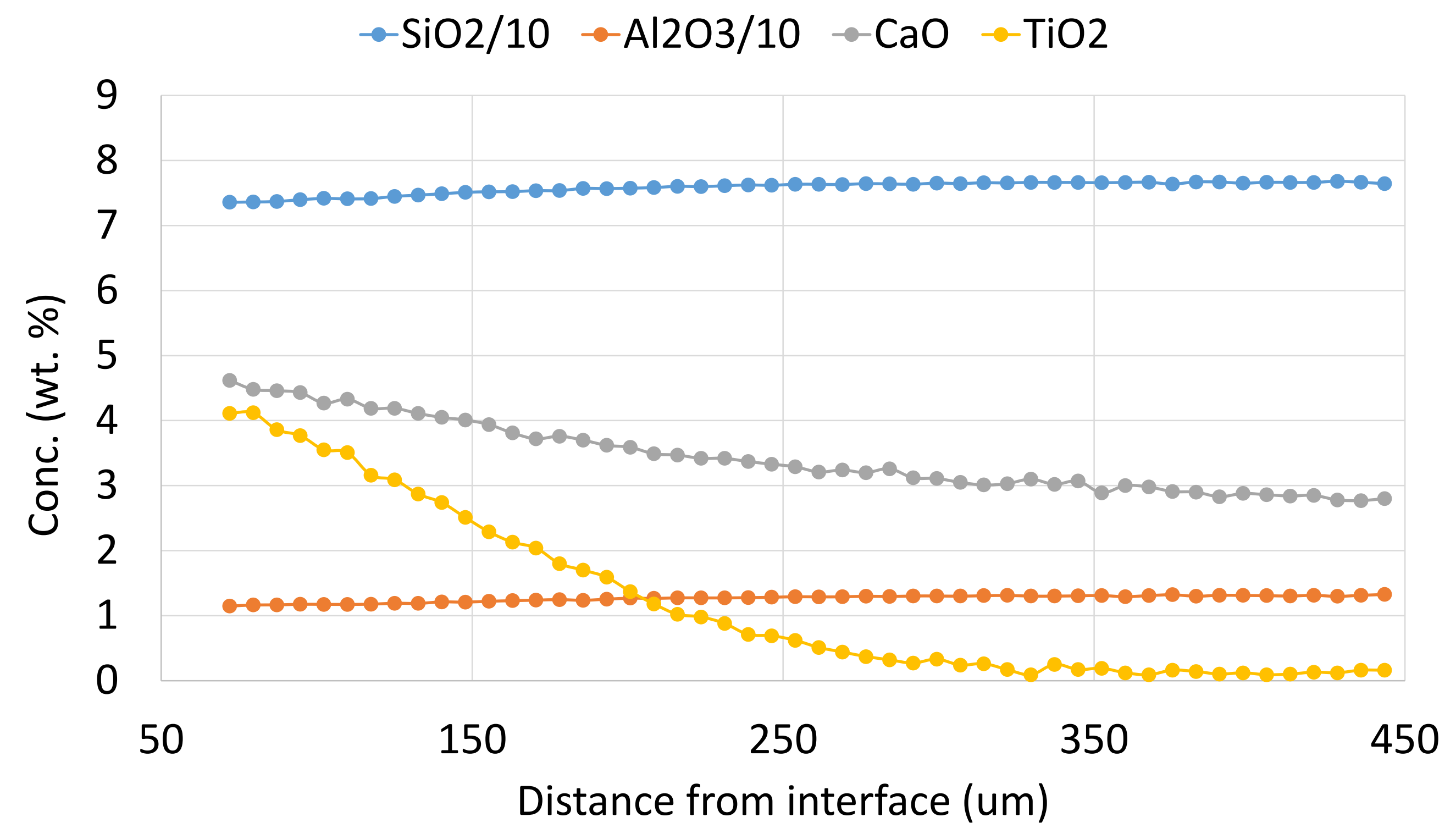




\section{Diffusion profiles from dissolution experiments}

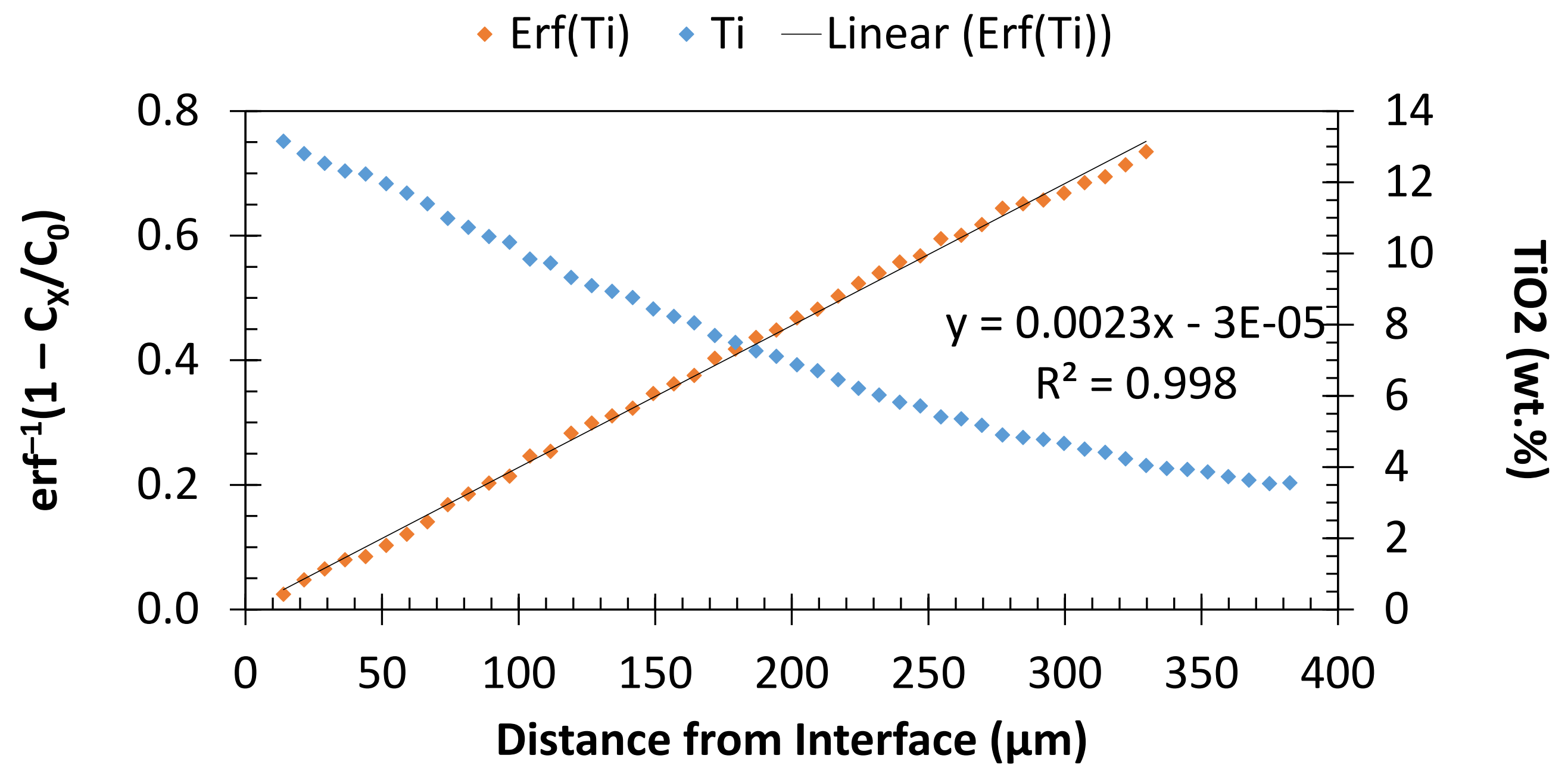

SphDis.3, $\mathrm{T}=1300^{\circ} \mathrm{C}, \mathrm{P}=0.8 \mathrm{GPa}, \mathrm{H}_{2} \mathrm{O}=1.9$ wt. $\%$, duration $=18 \mathrm{~h}$ 
Comparison of growth

- Growth - Dissolution and

dissolution experiment results

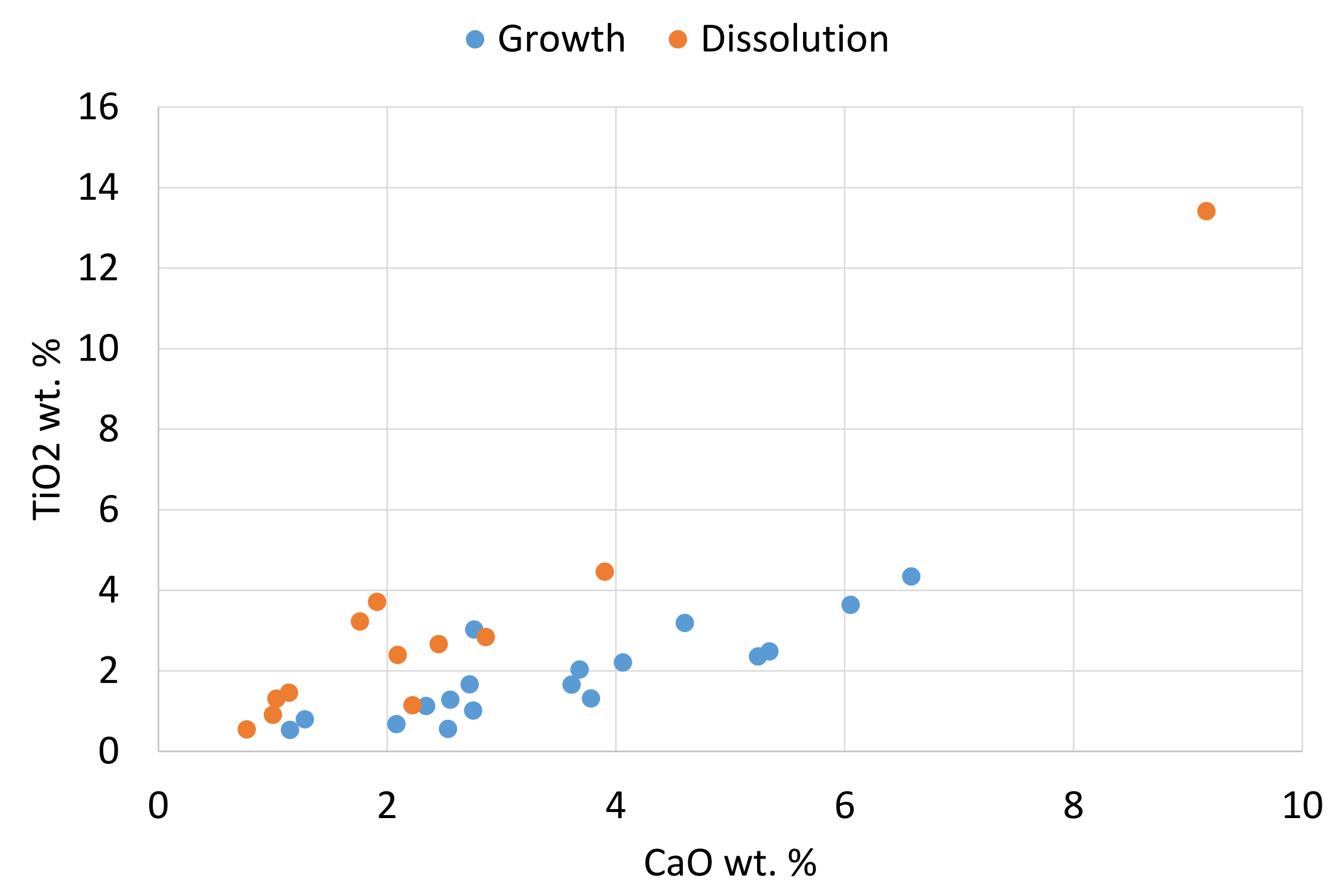




\section{Solubility Equation}

$$
\mathrm{CaTiSiO}_{5} \text { sphene }=\mathrm{CaO}^{\text {melt }}+\mathrm{TiO}_{2}^{\text {melt }}+\mathrm{SiO}_{2}^{\text {melt }}
$$

- The activity of $\mathrm{CaTiSiO}_{5}$ in sphene $\sim 1$. Thus, In $\mathrm{K}=\ln [\mathrm{CaO}]^{\text {melt }}+\ln \left[\mathrm{TiO}_{2}\right]^{\text {melt }}+\ln \left[\mathrm{SiO}_{2}\right]^{\text {melt }}=-\Delta \mathrm{G} / \mathrm{RT}$ $\ln \mathrm{C}_{\mathrm{TiO} 2}{ }^{\text {melt }}=-\Delta \mathrm{H} / \mathrm{RT}+\Delta \mathrm{S} / \mathrm{R}-\Delta \mathrm{V}(\mathrm{P}-1) / \mathrm{RT}-\ln \mathrm{T}_{\mathrm{TiO} 2}$ melt $-\ln [\mathrm{CaO}]-\ln \left[\mathrm{SiO}_{2}\right]$

- The last three terms are replaced by a single melt composition parameter $\mathrm{C}=(10 * \mathrm{eCa}) /(\mathrm{Al} * \mathrm{Si})$, symbols are cation fractions, and excess calcium eCa $=\mathrm{CaO}-\mathrm{Al}_{2} \mathrm{O}_{3}+\mathrm{Na}_{2} \mathrm{O}+\mathrm{K}_{2} \mathrm{O}$ with concentrations in moles

- Multiple linear regression of glass composition data from 23 growth and dissolution experiments yielded the sphene solubility equation (adj. $r=$ 0.97):

$\mathrm{TiO}_{2}($ wt. $\%)=0.79 * \mathrm{C}-7993 / \mathrm{T}(\mathrm{K})+7.88$ 


\section{Temperature dependence of sphene solubility}

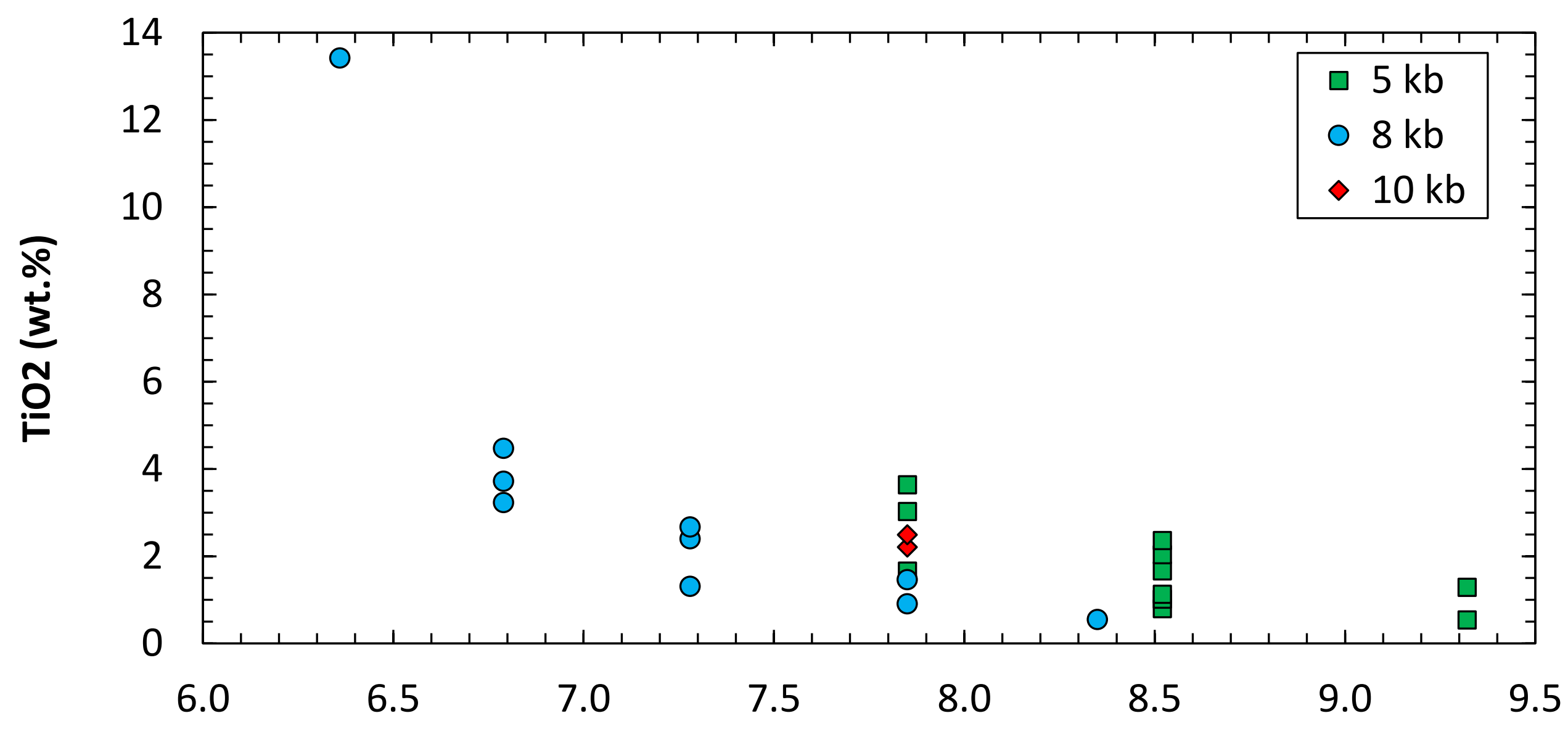

10,000/Temperature (K-1) 
Dependence of sphene solubility on melt composition parameter $\mathrm{C}$ and $\mathrm{T}$

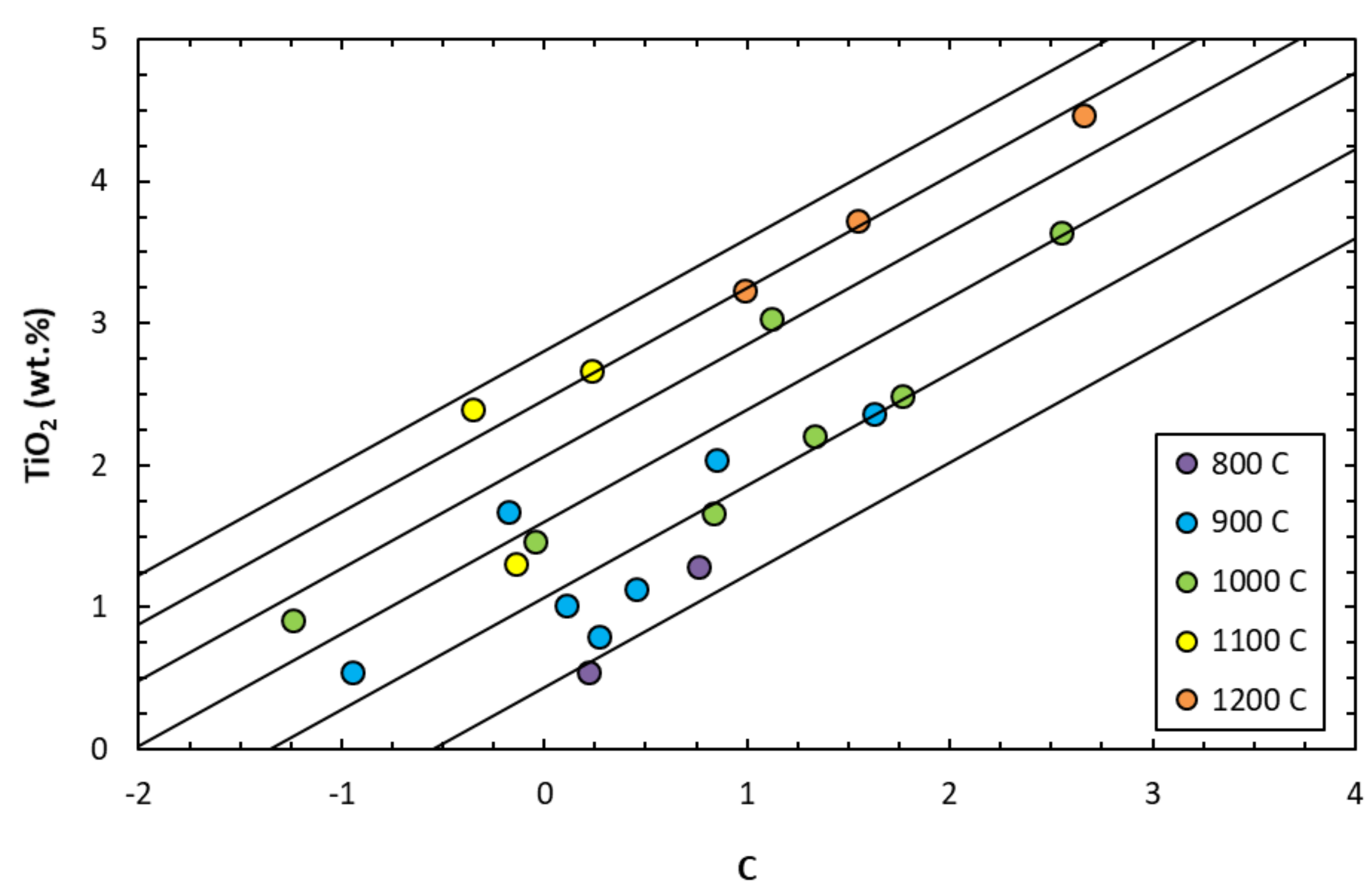

$\mathrm{C}=(10 * \mathrm{eCa}) /(\mathrm{Al} * \mathrm{Si})$ and excess $\mathrm{Ca}$ eCa $=\mathrm{CaO}-\mathrm{Al}_{2} \mathrm{O}_{3}+\mathrm{Na}_{2} \mathrm{O}+\mathrm{K}_{2} \mathrm{O}$ with concentrations in moles 


\section{Comparison to Sphene-bearing Natural Samples}

\begin{tabular}{|c|c|c|c|c|c|}
\hline Sample Name & Location $^{f}$ & $\begin{array}{l}\text { Sample } \\
\text { Type }^{a}\end{array}$ & $\left|\begin{array}{c}\text { Zir. Sat. } \mathrm{T}\left({ }^{\circ} \mathrm{C}\right) \\
\mathrm{W}+\mathrm{H}^{\mathrm{b}}\end{array}\right|$ & $\begin{array}{l}\text { Sphene Sat. } \\
\text { Temp. }\left({ }^{\circ} \mathrm{C}\right)^{\mathrm{C}}\end{array}$ & Remarks $^{\mathrm{e}}$ \\
\hline FCT intracaldera & Fish Canyon Tuff & glass & - & 775 & Fe-Ti oxide temp $=760 \pm 30{ }^{\circ} \mathrm{C}$ \\
\hline FCT outflow & Fish Canyon Tuff & glass & - & 805 & Fe-Ti oxide temp $=760 \pm 30{ }^{\circ} \mathrm{C}$ \\
\hline HRL16 & Highland Range & glass & 712 & 783 & Zr-in-sphene T mostly $710-740{ }^{\circ} \mathrm{C}$ \\
\hline HRL21 & Highland Range & glass & 734 & 787 & Zr-in-sphene T mostly $710-740{ }^{\circ} \mathrm{C}$ \\
\hline KPST01 d & Peach Spring Tuff & WR & 809 & 742 & Avg Zr-in-sph temps $770 \pm 20{ }^{\circ} \mathrm{C}$ \\
\hline PSTG ${ }^{d}$ & Peach Spring Tuff & WR & 901 & 819 & Avg Zr-in-sph temps $770 \pm 20^{\circ} \mathrm{C}$ \\
\hline WSW2 ${ }^{d}$ & Peach Spring Tuff & WR & 833 & 753 & Avg Zr-in-sph temps $770 \pm 20^{\circ} \mathrm{C}$ \\
\hline \multicolumn{6}{|c|}{$\begin{array}{l}\text { a glass }=\text { matrix glass, } W R=\text { whole-rock. } \\
\text { b Zircon solubility models: } W+\mathrm{H}-\text { Watson and Harrison (1983), B et. al.-Boehnke et al. (2013). The Watson and Harrison temperatures are preferred due to } \\
\text { the low resolution of the Boehnke et al. model at low temperatures. }\end{array}$} \\
\hline \multicolumn{6}{|c|}{${ }^{\mathrm{c}}$ Solubility model of this study. } \\
\hline \multicolumn{6}{|c|}{$\begin{array}{l}\text { d The saturation temperature is an average of those calculated for each individual analysis and is not calculated from the average composition. } \\
\text { e Zr-in-sphene thermometer of Hayden et al. (2008). } \\
\text { f Bachmann et al (2002) for Fish Canyon Tuff, Colombini et al (2011) for Highland Range, Pamukcu et al (2013) for Peach Springs Tuff }\end{array}$} \\
\hline
\end{tabular}




\section{Conclusions}

- Sphene solubility in siliceous melts increases with increasing $\mathrm{CaO} /$ metaluminosity and increasing temperature.

- For the compositions and conditions studied, sphene solubility does not depend significantly on melt $\mathrm{H}_{2} \mathrm{O}$ concentration or pressure.

- Results will be verified through reanalysis using an electron microprobe.

-We plan to compare sphene saturation temperatures calculated for natural samples to temperatures calculated using other thermometers. 